\title{
ESTUDO QUALITATIVO DA FORMAÇÃO HIPOCAMPAL DE ANIMAIS HIPERTENSOS COM EPILEPSIA
}

\author{
Fulvio Alexandre Scorza1, Ricardo Mario Arida², Roberta Monterazzo Cysneiros³, \\ Carla Alessandra Scorza4, Marly de Albuquerque5, Esper Abrão Cavalheiro6
}

\begin{abstract}
RESUMO - O objetivo deste estudo foi avaliar qualitativamente o hipocampo e o giro dentado de ratos espontaneamente hipertensos (SHR) com epilepsia. Método: Os animais foram divididos em 4 grupos: Wistar controle, Wistar com epilepsia, SHR controle e SHR com epilepsia. Para indução da epilepsia, utilizamos o modelo da pilocarpina. Após os animais apresentarem crises espontâneas e recorrentes, o tecido cerebral dos animais foi encaminhado para análise histológica através dos métodos de Nissl e neo-Timm. Resultados: Nos animais Wistar e SHR controle submetidos à coloração de Nissl observamos a manutenção das camadas celulares da formação hipocampal. Nos animais Wistar com epilepsia verificamos intensa morte neuronal na região CA1 e CA3 do hipocampo e no hilo do giro dentado. Nos animais SHR com epilepsia verificouse a presença de atrofia hipocampal com dilatação do sistema ventricular. A coloração de neo-Timm revelou a presença de brotamento supragranular em todos os animais com epilepsia. Conclusão: Foram encontradas alterações neuropatológicas na citoarquitetura hipocampal nos animais Wistar com epilepsia e SHR com epilepsia, demonstrando que a epilepsia isoladamente ou associadamente à hipertensão são capazes de causar destruição neuronal.
\end{abstract}

PALAVRAS-CHAVE: epilepsia, hipertensão arterial, crise epiléptica, pilocarpina.

\begin{abstract}
Qualitative study of hippocampal formation in hypertensive rats with epilepsy.
ABSTRACT - The aim of our study was to investigate the hippocampus and dentate gyrus neuropathological features of spontaneous hypertensive rats (SHR) with epilepsy. Method: Animals were randomly divided into 4 groups: control Wistar, Wistar with epilepsy, control SHR and SHR with epilepsy. The pilocarpine model of epilepsy was used in this experiement. After spontaneous recurrent seizures, all animals were perfused and their brains processed for histological analysis through Nissl and neo-Timm methods. Results: In the Wistar rats with epilepsy we observed cell loss in hippocampal subfields CA1, CA3 and hilus of the dentate gyrus when compared with control animals. In the SHR with epilepsy we observed hippocampal formation atrophy with ventricular dilatation. No morphological alterations were observed in SHR and Wistar control rats. The neo-Timm staining of hippocampal formation has shown supragranular sprouting in Wistar and SHR with epilepsy. Conclusion: We found neuropathological alterations in hippocampal formation in Wistar with epilepsy and SHR with epilepsy, suggesting that epilepsy per se or associated to hypertention are able to cause neuronal damage.
\end{abstract}

KEY WORDS: epilepsy, arterial hypertension, seizure, pilocarpine.

A forma mais comum de síndrome epiléptica é a epilepsia do lobo temporal' ${ }^{1}$, caracterizada pela presença de crises parciais complexas com início nas estruturas límbicas, as quais incluem o hipocampo e o giro dentado². A formação hipocampal compreende quatro regiões corticais: o giro dentado, o hi- pocampo propriamente dito, o complexo subicular e o córtex entorrinal. $O$ giro dentado é dividido em três camadas: a camada molecular, camada de células granulares e camada polimórfica (hilo). Já o hipocampo propriamente dito é dividido em três subcampos: CA3, CA2 e CA1. Nesse sentido, os cir-

\footnotetext{
Disciplina de Neurologia Experimental da Universidade Federal de São Paulo/Escola Paulista de Medicina, São Paulo SP (UNIFESP/EPM): ${ }^{1}$ Professor Adjunto Visitante da Disciplina de Neurologia Experimental UNIFESP/EPM,. Laboratório de Neurociências - Núcleo de Pesquisas Tecnológicas/Universidade de Mogi das Cruzes (NPT/UMC); ${ }^{2}$ Professor Adjunto Visitante da Disciplina de Neurologia Experimental UNIFESP/EPM. Laboratório de Neurociências NPT/UMC; ${ }^{3}$ Pesquisadora Associada do Laboratório de Neurologia Experimental UNIFESP/EPM, Professora Titular de Farmacologia do Centro Universitário São Camilo; ${ }^{4}$ Doutoranda da Disciplina de Neurologia Experimental UNIFESP/EPM. Mestre em Neurociências UNIFESP/EPM; ${ }^{5}$ Professora Adjunta de Neurologia da Faculdade de Medicina da Universidade de Mogi das Cruzes. Pesquisadora Associada do Laboratório de Neurociências NPT/UMC; ${ }^{6}$ Professor Titular da Disciplina de Neurologia Experimental.
}

Recebido 5 Julho 2004, recebido na forma final 22 Setembro 2004. Aceito 13 Novembro 2004. 
cuitos anatômicos da formação hipocampal incluem a clássica via tris-sináptica: o giro dentado recebe sua maior aferência do córtex entorrinal (via perfurante); as células granulares projetam através das fibras musgosas para a região hilar e subcampo CA3. Das células piramidais de CA 1 emergem colaterais axônicos que terminam em neurônios de CA3 vias conexões de associação e também proporcionam a maior aferência para os neurônios piramidais de CA1 (via colateral de Schaffer) ${ }^{3}$.

A importância clínica da epilepsia do lobo temporal deve-se à sua alta prevalência e elevada proporção de pacientes que não respondem aos diferentes esquemas terapêuticos, e que tem bom prognóstico com o tratamento cirúrgico, com $80 \%$ a $90 \%$ de probabilidade de controle de crises $^{4}$. Dessa maneira, uma vez que as epilepsias humanas apresentam crises recorrentes e na maioria das vezes ocorrem de forma espontânea, os modelos crônicos de epilepsia se revestem de maior importância por mimetizarem mais adequadamente a condição humana servindo, portanto, para maior caracterização fisiopatológica e para avaliação de drogas antiepilépticas ${ }^{5,6}$. Dentre esses modelos, o da pilocarpina tem sido amplamente usado em função da facilidade técnica e, principalmente, pela semelhança fisiopatológica com a epilepsia do lobo temporal em seres humanos? ${ }^{7}$.

O seguimento temporal do modelo da pilocarpina permitiu dividi-lo em três fases: período agudo, no qual os animais apresentam as crises límbicas evoluindo para status epilepticus; período silencioso, com duração em torno de 4 a 44 dias (média de 14,8 dias e mediana de 10 dias) caracterizado pela ausência de sinais comportamentais e eletrográficos de atividade epiléptica; e período crônico, que se inicia com a primeira crise espontânea que recorre durante toda a vida do animal ${ }^{8}$. Essas crises espontâneas se iniciam com automatismos faciais, clonias dos membros anteriores, elevação do corpo com apoio nas patas posteriores e perda do equilíbrio seguida por crise clônica generalizada, com duração aproximada de 40 a 60 segundos $^{8}$. Do ponto de vista estrutural, o hipocampo é a região cerebral mais sensível aos efeitos neuropatológicos desencadeados pela ação epileptiforme resultante da ativação colinérgica ${ }^{7,9}$.

A hipertensão arterial é definida como uma elevação da pressão sangüínea arterial acima de $140 / 90 \mathrm{mmHg}^{10}$ e aproximadamente $24 \%$ da população adulta dos Estados Unidos tem esse diagnóstico ${ }^{11}$. A hipertensão está diretamente relacionada com o surgimento de várias complicações cardiovas- culares, dentre elas o acidente vascular cerebral e o infarto do miocárdio ${ }^{12}$.

Para melhor compreensão da fisiopatologia e da terapêutica da hipertensão arterial, Okamoto e Aoki desenvolveram, por seleção genética, uma linhagem de ratos espontaneamente hipertensos $(\mathrm{SHR})^{13}$. Ao nascer, os ratos SHR são normotensos, mas por volta da quinta semana de vida, a pressão sangüínea arterial eleva-se progressivamente, atingindo ou excedendo $220 \mathrm{mmHg}$, mantendo-se assim durante toda a vida do animal, tornando-se o modelo experimental de maior utilidade para o estudo fisiopatológico da hipertensão essencial humana ${ }^{14}$. A seleção da linhagem de ratos SHR foi concretizada por meio de sucessivos cruzamentos de casais da colônia Wistar, com tendência a pressão sangüínea elevada, oriundos do centro de animais da Kyoto University, em Kyoto, Japão.

A relação existente entre epilepsia e hipertensão ainda não está bem estabelecida, no entanto as crises epilépticas são comumente encontradas em pacientes portadores de doenças sistêmicas e dentre os fatores capazes de desencadear tais crises está a hipertensão, pois alterações na permeabilidade e integridade vascular resultantes da encefalopatia hipertensiva, podem levar a pequenas áreas de edema e hemorragia, sendo capazes de gerar crises $^{15}$. Paralelamente, a hipertensão é o principal fator de risco para o aparecimento de um acidente vascular cerebral e este, por sua vez, é importante fator de risco para o surgimento de crises epilépticas subseqüentes ${ }^{16}$. Em contrapartida, indivíduos portadores de hipertensão grave e incontrolável, na ausência de acidente vascular cerebral anterior, também apresentavam crises epilépticas ${ }^{17}$.

Como as epilepsias e a hipertensão arterial são doenças crônicas com alta incidência e prevalência na população brasileira, o objetivo do presente estudo, utilizando o modelo de epilepsia induzido pela pilocarpina, foi estudar a extensão das alterações histopatológicas hipocampais na vigência destas patologias.

\section{MÉTODO}

Animais - Para a realização deste estudo utilizamos ratos machos, da raça Wistar e SHR adultos, pesando entre 250 e $300 \mathrm{~g}$ no início dos experimentos. Os animais eram provenientes do Biotério Central da Universidade Federal de São Paulo/Escola Paulista de Medicina, e foram mantidos no biotério do laboratório da Disciplina de Neurologia Experimental durante todo o período de realização dos experimentos. Os animais foram alojados em grupos de cinco ratos, em gaiolas apropriadas, onde 
tinham livre acesso à comida e água. As condições do biotério obedecem a um ciclo claro-escuro de 12 horas (claro: 7:00h - 19:00h), sendo a temperatura ambiente mantida constante entre $21^{ \pm} 1{ }^{\circ} \mathrm{C}$. Os protocolos experimentais utilizados neste trabalho foram aprovados pelo Comitê de Ética em Pesquisa da Universidade Federal de São Paulo, processo 629/97.

Indução do modelo de epilepsia induzido pela pilocarpina - Para obtenção de animais com epilepsia foi utilizado o modelo da pilocarpina (Merck S.A.). No desenvolvimento deste estudo utilizou-se a dose de $350 \mathrm{mg} / \mathrm{kg}$ para todos os animais, isto é, Wistar e SHR. Esta droga foi injetada intraperitonealmente e os seus efeitos foram observados e classificados segundo a metodologia descrita por Cavalheiro ${ }^{7}$. Para a indução do status epilepticus utilizamos um total de 20 animais, sendo 10 Wistar e 10 SHR. Todos os animais injetados com pilocarpina foram pré-tratados subcutaneamente com metil-escopolamina $1 \mathrm{mg} / \mathrm{kg}$ (Sigma Chemica CO), 30 minutos antes de injeção da pilocarpina. A administração da metil-escopolamina tinha por finalidade atenuar os efeitos periféricos provocados pela injeção de pilocarpina. Os animais pertencentes ao grupo controle (SHR $n=6$; Wistar $n=6$ ) também receberam pré-tratamento com metil-escopolamina ( $1 \mathrm{mg} / \mathrm{kg}) ;$ no entanto, receberam solução salina $0,9 \%$ ao invés de pilocarpina.

Os animais sobreviventes ao status epilepticus (SHR $n=6$; Wistar $n=6$ ), foram acondicionados individualmente em cilindros de acrílico transparentes em uma sala com sistema de monitoramento de vídeo (24h/dia; Philips) para detecção de crises epilépticas espontâneas e recorrentes.

Análise histológica - Após os animais apresentarem crises espontâneas e recorrentes, foram perfundidos e os cérebros retirados para posterior análise histológica através do método de neo-Timm e Niss ${ }^{9}$. O método de neo-Timm intensifica a coloração específica de zinco, produzindo melhor visualização das fibras musgosas, permitindo fácil observação da reorganização sináptica. 0 método de Nissl utiliza o cresil violeta, um corante básico utilizado para evidenciar o citoplasma de neurônios. O citoplasma contém várias estruturas, inclusive os corpúsculos de Nissl (ou substância de Nissl). Esses corpúsculos são compostos, basicamente, por RNA. Dessa maneira, tal coloração serve como indicador da viabilidade neuronal pois, quando da ocorrência de lesão neuronal, esses corpúsculos podem desaparecer (fenômeno conhecido por cromatólise).

Para a perfusão, os animais foram anestesiados profundamente com tionembutal 2,5\% (Abbott). Na primeira fase da perfusão os animais receberam uma solução de sulfeto de sódio 0,1\% (Labsynth Produtos para Laboratório Ltda.), e em seguida uma fixadora de glutaraldeído 3\% (Merck S.A.). Todas as soluções foram preparadas em tampão fosfato e o pH mantido em 7,4. A infusão foi feita automaticamente por uma bomba de perfusão (Materflex). Em seguida, os cérebros foram retirados da caixa craniana e colocados na solução crioprotetora de sacarose $30 \%$ (Merck S.A.). Os cérebros foram, então, cortados de forma seriada em secções de $40 \mu \mathrm{m}$ de espessura em micrótomo de congelação (Leika, modelo Jung CM 1800).

\section{RESULTADOS}

De acordo com o modelo de epilepsia límbica desenvolvido neste estudo, a presença de status epilepticus ocorreu após a administração i.p. de pilocarpina (350 mg/kg) e todos os animais que sobreviveram ao estado de mal epiléptico desenvolveram crises espontâneas e recorrentes.

O estado de mal epiléptico teve duração de aproximadamente 8 horas, quando os animais tornavam-se não responsivos aos estímulos ambientais, retornando gradativamente a seu comportamento normal dentro de 24 horas, tanto nos animais Wistar quanto nos SHR. O período silencioso teve uma duração média de 16 dias. É importante salientar que não foram observadas diferenças quanto a duração do período silencioso entre os ratos Wistar e SHR. A crise espontânea e recorrente dos animais Wistar e SHR foi caracterizada pelo aparecimento de movimentos mastigatórios, piscamento e eventual salivação seguida por abalos clônicos de um ou dos dois membros anteriores. A esse comportamento se segue elevação do tronco sobre as patas posteriores concomitantemente a clonias das patas anteriores, para finalmente perder o equilíbrio postural e apresentar convulsão tônico-clônica generalizada. Esta seqüência de eventos durou aproximadamente 60 segundos.

Estudo histológico - Nos cortes histológicos dos cérebros dos animais Wistar pertencentes ao grupo controle submetidos à coloração de Nissl observamos a manutenção das camadas celulares em todas as regiões do hipocampo, bem como na região do hilo do giro dentado (Fig 1A). Em contrapartida, nos animais Wistar com epilepsia pudemos observar uma intensa morte neuronal predominando na região CA1 e CA3 do hipocampo (Fig 1B).

Paralelamente, não foram observadas alterações morfológicas nos cortes histológicos cerebrais dos animais SHR controle (Fig 1C). Já o estudo histológico dos cortes cerebrais dos animais SHR com epilepsia revelou a presença de atrofia hipocampal com profundo desarranjo das camadas celulares nessa região e importante dilatação do sistema ventricular (Fig 1D).

A coloração de neo-Timm (Figs 2A, B, C, D) revelou a presença de brotamento supragranular em 


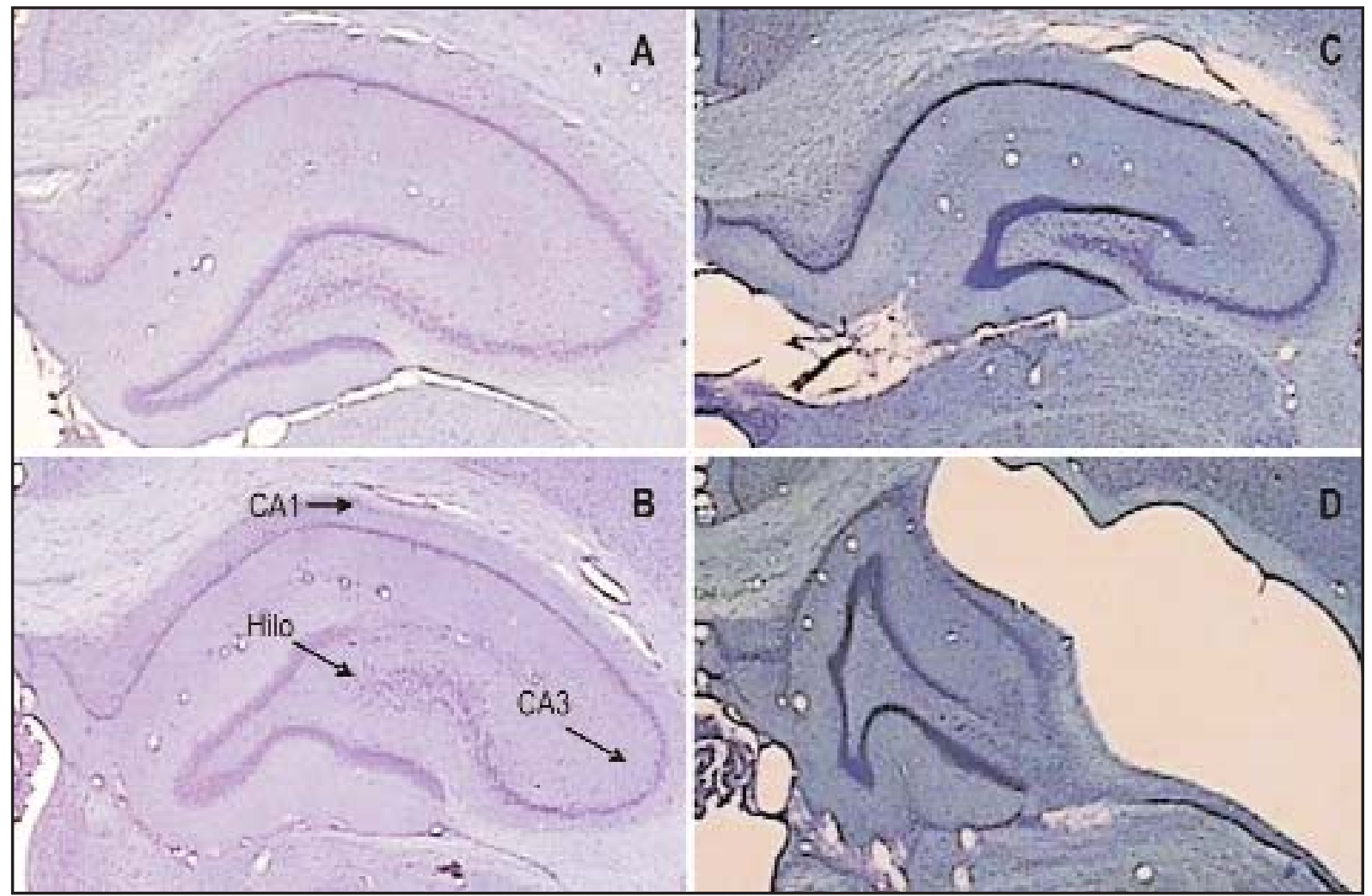

Fig 1. Fotografia de corte coronal do cérebro de ratos no nível de hipocampo corado pela técnica de Nissl. (A) Wistar controle, (B) Wistar com epilepsia, (C) SHR controle, (D) SHR com epilepsia.

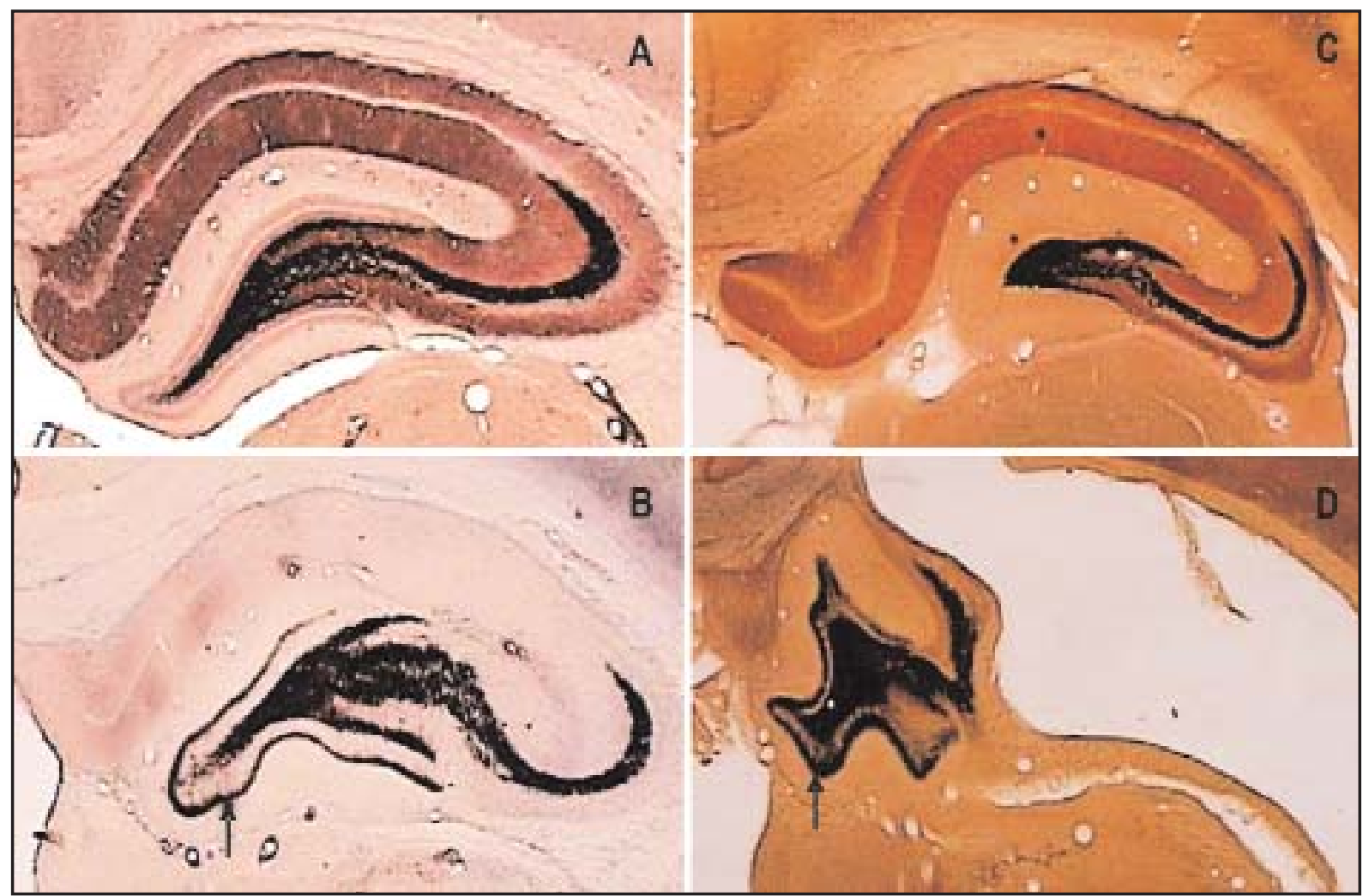

Fig 2. Fotografia de corte coronal do cérebro de ratos no nível de hipocampo corado pela técnica de neo-Timm. (A) Wistar controle, (B) Wistar com epilepsia, (C) SHR controle, (D) SHR com epilepsia. 
todos os animais que receberam pilocarpina e apresentaram status epilepticus, tanto nos somente Wistar com de epilepsia (Fig 2B), bem como nos SHR com epilepsia (Fig 2D).

\section{DISCUSSÃO}

Estudos utilizando modelos experimentais de epilepsia vêm fornecendo, nas últimas décadas, importantes avanços para a compreensão dos mecanismos envolvidos na epilepsia humana. Nossos resultados revelaram que os animais Wistar com epilepsia apresentaram intensa morte neuronal predominando na região CA1 e CA3 do hipocampo. Já nos animais SHR com epilepsia notamos importante atrofia hipocampal acompanhada de um desarranjo das camadas celulares nessa região e importante dilatação do sistema ventricular. Todos os animais que apresentaram crises espontâneas e recorrentes mostraram a presença de brotamento supragranular, isto é, brotamento de axônios dos neurônios da camada granular para os neurônios da camada molecular interna do giro dentado?

A indução do estado de mal epiléptico pela pilocarpina produz alterações cerebrais difusas. Estas lesões resultam em alterações neuropatológicas permanentes, detectáveis no córtex olfatório, complexo amigdalóide, tálamo, substância negra, formação hipocampal e neocórtex ${ }^{18}$. Dentre estas estruturas, o hipocampo é a região cerebral mais sensível aos efeitos neuropatológicos desencadeados pela ação epileptiforme resultante da ação colinérgica. Intensa destruição neuronal é observável em ambas as regiões do hipocampo (dorsal e ventral), principalmente na sub-área $\mathrm{CA} 3^{19}$. Essa perda neuronal parece relacionar-se com a liberação de aminoácidos excitatórios e sua interação com a membrana dos elementos pós-sinápticos vulneráveis, pois na lesão epiléptica, a ativação dos canais de $\mathrm{Ca}^{++}$pelos aminoácidos excitatórios determina seu influxo nos elementos celulares com alta densidade de canais de $\mathrm{Ca}^{++}$, alterando a homeostase intracelular e promovendo a lipólise e proteólise ${ }^{20}$. Nesse sentido, este fenômeno explica de forma pertinente a lesão celular na formação hipocampal encontrada nos animais Wistar e SHR submetidos ao status epilepticus em nosso estudo. Paralelamente, além de extensa degeneração neuronal, todos os animais com epilepsia em nosso estudo apresentaram brotamento supragranular, isto é, brotamento de axônios dos neurônios da camada granular para os neurônios da camada molecular interna do giro dentado9 ${ }^{9}$. Este padrão clássico de plasticidade neuronal explica o aparecimento de crises espontâneas e recorrentes nos animais Wistar e SHR com epilepsia.

Além dos aspectos relatados anteriormente, a perda neuronal presente nos animais SHR observada em nosso estudo também pode ser reflexo de um processo neurodegenerativo. Sendo assim, dentre os mecanismos fisiológicos envolvidos com este processo, a progressiva deterioração da homeostase intracelular de cálcio tem recebido especial atenção, pois alterações dessa homeostase levam a sérias conseqüências no processo de excitação neuronal e de comunicação celular ${ }^{21,22}$. Paralelamente, a relação entre hipertensão, condição cerebrovascular e funcionamento neuronal no sistema nervoso central (SNC) tem sido demonstrada em diversos estudos, sugerindo que a hipertensão acelera as alterações neuronais e comportamentais quando comparada com animais normotensos ${ }^{23}$. Nesse sentido, Luiten e colaboradores ${ }^{24}$ demonstraram que a hipertensão e o processo neurodegenerativo devem exercer um profundo impacto sobre a estrutura da parede vascular cerebral, tanto nos pequenos quanto nos grandes vasos, sugerindo que a disfunção das células endoteliais é o maior fator causal para o declínio acelerado do funcionamento do SNC em ratos SHR durante o processo neurodegenerativo.

Outras duas hipóteses ainda podem ser especuladas. A primeira delas estaria relacionada aos fatores de crescimento fibroblástico (bFGF), agentes neurotróficos que desempenham importante papel sobre o crescimento, diferenciação e sobrevida neuronal ${ }^{24,25}$. Demonstrou-se também que não há variação da expressão de bFGF na região hipocampal de animais SHR quando comparado com animais normotensos ${ }^{26}$. Sendo assim, a normatização da expressão hipocampal desses agentes neurotróficos (bFGF) nos animais hipertensos com epilepsia poderia explicar o processo degenerativo existente nessa região cerebral em nosso estudo. A segunda hipótese poderia estar relacionada com o processo de neurogênese. Dentre todas as espécies de mamíferos já estudadas, existem duas regiões onde se localizam células progenitoras mitoticamente ativas, capazes de gerar novos neurônios durante a fase adulta ${ }^{27,28}$. Essas regiões incluem os ventrículos, mais precisamente a zona subventricular dos ventrículos laterais (ZSV) e a formação hipocampal, especificamente a zona subgranular do giro denteado (ZSG), sendo esta última, o ponto de encontro da camada interna de células granulares do giro denteado com o hilo. Nesse sentido, Perfilieva e colabo- 
radores ${ }^{29}$ demonstraram aumento da atividade mitótica na região do giro dentado de animais SHR quando comparado com animais normotensos. Surpreendentemente, a possível existência de proliferação celular na formação hipocampal dos animais SHR com epilepsia em nosso estudo não foi capaz de impedir ou reverter a perda e desorganização celular nessa região. Algumas possibilidades poderiam explicar esse fenômeno: primeiro, o número de células lesadas deve ser quantitativamente maior em relação às novas células geradas; segundo, os neurônios gerados provavelmente não se tornam suficientemente maduros e portanto, não são neurofisiologicamente funcionais e, finalmente, o ambiente cerebral dos animais SHR com epilepsia pode ser tóxico para os novos neurônios.

Em conclusão, foram encontradas alterações neuropatológicas na citoarquitetura hipocampal nos animais Wistar com epilepsia e SHR com epilepsia, demonstrando que a epilepsia isoladamente ou associadamente à hipertensão são capazes de causar destruição neuronal. Obviamente, muitas questões ainda devem ser respondidas; no entanto, evidências clínicas sugerem que a ocorrência concomitante da hipertensão arterial com a epilepsia altera a freqüência de crises epilépticas. Por outro lado, cabe ressaltar que, a longo prazo, a hipertensão arterial pode resultar em lesões do SNC, facilitando tanto a epileptogênese pela própria lesão em si quanto pela desorganização morfofuncional das estruturas cerebrais. Sendo assim, futuros estudos deverão ser desenvolvidos na tentativa de elucidação dessas questões.

\section{REFERÊNCIAS}

1. Hauser WA, Kurland LT. The epidemiology of epilepsy in Rochester, Minnesota, 1935 though 1937. Epilepsia 1975;16:1-66.

2. Sutula T, He XX, Cavazos J, Scott G. Synaptic reorganization in the hippocampus induced by abnormal functional activity Science 1998; 239:1147-1150.

3. Leite JP, Cavalheiro EA. Neurobiologia da esclerose medial temporal. In Costa JC, Palmini A, Yacubian EMT, Cavalheiro EA (eds) Fundamentos neurobiológicos das epilepsias: aspectos clínicos e cirúrgicos: São Paulo: Lemos Editorial, 1998;129-136.

4. Paglioli-Neto E, Cendes F. Tratamento cirúrgico: In Guerreiro CA, Guerreiro MM, Cendes F, Lopes-Cendes (eds). Epilepsia. São Paulo: Lemos Editorial, 2000;379-393.

5. Leite JP, Bortolotto ZA, Cavalheiro EA. Spontaneous recurrent seizures in rats: an experimental model of partial epilepsy. Neurosci Behav Rev 1990;14:511-517.
6. Blumcke I, Beck H, Lie AA, Wiestler D. Molecular neuropathology of human mesial temporal lobe epilepsy. Epilepsy Res 1999;36:205-223.

7. Cavalheiro EA. The pilocarpine model of epilepsy. Ital J Neurol Sci 1995; 16:33-37.

8. Cavalheiro EA, Leite JP, Bortolotto ZA, Turski WA, Ikonomidou C, Turski L. Long-term effect of pilocarpine in rats:structural damage of the brain triggers kindling and spontaneously recurrent seizures Epilepsia 1991;32:778-782.

9. Mello LEAM, Cavalheiro EA, Tan AM, et al. Circuit mechanisms of seizures in the pilocarpine model of chronic epilepsy: cell loss and mossy fiber sprouting. Epilepsia 1993;34:985-995.

10. Hardman TC, Lunnon MW. The current status of antihypertensive treatments: into the new millennium. Expert Opin Pharmacother 2001; 2:731-737.

11. Burt VL, Whelton P, Rocella EJ, et al. Prevalence of hypertension in the US adult population: results from the Third National Health and Nutrition Examination Survey, 1988-1991.Hypertension 1995;25:305-313.

12. Cushman WC. Systolic hypertension and cardiovascular risk reduction: a clinical review. Curr Hypertens Rep 2001;Suppl1:S11-S15.

13. Okamoto K, Aoki K. Development of a strain of spontaneous hypertensive rats. Jap Circ J 1963;27:282-293.

14. Institute of Laboratory Animal Resources (ILAR). Spontaneous hypertensive rats: guidelines for breeding, care and use. In Committee on care and use of spontaneous hypertensive rats (SHR) 1976;19(3):G1-G20.

15. Delanty N, Vaughan C, French J. Medical causes of seizures. Lancet 1998; 352:383-390.

16. Ng SKC, Hauser WA, Brust JCM, Susser M. Hypertension and the risk of new-onset unprovoked seizures. Neurology 1993;43:425-428.

17. Hesdorffer DC, Hauser WA, Annergers F, Rocca WA. Severe uncontrolled hypertension and adult-onset seizures: a case control study in Rochester, Minnesota. Epilepsia 1996;37:736-741.

18. Turski WA, Cavalheiro EA, Bortolotto ZA, Mello LEAM, Schwarz M, Turski L. Seizures produced by pilocarpine in mice: a behavioral, electroencephalographic and morphological analysis. Brain Res 1984;321:237-253.

19. Turski WA, Cavalheiro EA, Schwarz M, Czuczwar SJ, Kleinrok Z, Turski L. Limbic seizures produced by pilocarpine in rats: behavioural, electroencephalographic and neuropathological study. Behav Brain Res 1983;9:315-335.

20. Siesjo BK, Ingvar M, Wieloch T. Cellular and molecular events underlying epileptic brain damage. Ann N Y Acad Sci 1986;462:207-223.

21. Landfield PW, Campbell LW, Hao SY, Kerr DS. Aging-related increases in voltage-sensitive, inactivating calcium currents in rat hippocampus. Implications for mechanisms of brain aging and Alzheimer's disease. Ann N Y Acad Sci. 1989;568: 95-105.

22. Moyer JR Jr, Thompson LT, Black JP, Disterhoft JF. Nimodipine increases excitability of rabbit CA1 pyramidal neurons in an age- and concentration-dependent manner. J Neurophysiol 1992;68:2100-2109.

23. Wyss JM, Fisk G, van Groen T. Impaired learning and memory in mature spontaneously hypertensive rats. Brain Res 1992;592:135-140.

24. Luiten PG, de Jong GI, Schuurman T. Cerebrovascular, neuronal, and behavioral effects of long-term Ca2+ channel blockade in aging normotensive and hypertensive rat strains. Ann N Y Acad Sci 1994;747:431-451.

25. Gensburger C, Labourdette G, Sensenbrenner M. Brain basic fibroblast growth factor stimulates the proliferation of rat neuronal precursor cells in vitro. FEBS Lett 1987;217:1-5.

26. Lin TN, Wong YP, Chen JJ, et al. Elevated basic fibroblast growth factor levels in stroke-prone spontaneously hypertensive rats. Neuroscience 1997;76:557-570.

27. Gage F. Mammalian neural stem cells. Science 2000;287:1433-1438.

28. Gould E, Reeves AJ, Fallah M, Tanapat P, Gross CG, Fuchs E. Hippocampal neurogenesis in adult old world primates. PNAS 1999;96:5263-5267.

29. Perfilieva E, Risedal A, Nyberg J, Johansson BB, Eriksson PS. Gender and strain influence on neurogenesis in dentate gyrus of young rats. J Cereb Blood Flow Metab. 2001;21:211-217. 